\title{
Compiling Messages from Neutron Stars
}

\author{
The combination of gravitational-wave and $\mathrm{x}$-ray observations of neutron \\ stars provides new insight into the structure of these stars, as well as new \\ confirmation of Einstein's theory of gravity.
}

\author{
By Daniela Doneva
}

N eutron stars are some of the most compact objects known to exist in the Universe, second only to black holes. Their enormous densities produce strong gravitational fields and a large curvature in the surrounding spacetime. We would like to test gravity under these conditions, but we lack understanding of how matter behaves at such extreme densities. Specifically, we don't know the nuclear-matter equation of state, which-in its simplest form-relates the matter density to the pressure. A possible way to circumvent this limitation is to use universal relations connecting various neutron star properties in a way that is independent of the equation of state [1, 2]. Hector Silva from the Max Planck Institute for Gravitational Physics in Germany and his collaborators at the University of Illinois at Urbana-Champaign used such relations to infer, from recent $x$-ray observations, the moment of inertia, the quadrupole moment, and the surface eccentricity of an isolated neutron star [3]. By combining these mass-distribution estimates with gravitational-wave observations of merging neutron stars, they demonstrated a powerful way to constrain the strong-field regime of gravity. In particular, this "multimessenger" analysis of neutron star data provides new limits on parity symmetry violation in the gravitational interaction.

Observations of neutron stars can help us answer a long-standing question: Is Einstein's general relativity the correct theory of gravity in the regime of strong fields? Many theoretical considerations in fundamental physics, related to quantizing gravity or unifying all fundamental interactions, involve modifications of general relativity. Some alternative theories predict, for example, that gravitational interactions would, like the weak interactions, exhibit a parity asymmetry (or "handedness"). While researchers have tested Einstein's theory with remarkable accuracy for weak gravitational fields, probing the regime of large spacetime curvature was largely out of reach until the recent advances in gravitational-wave observations. Neutron stars, which have been observed through gravitational waves as well as with electromagnetic waves, are unique testbeds for Einstein's theory in this large curvature regime.

The density in the neutron star core reaches a few times $10^{15} \mathrm{~g} / \mathrm{cm}^{3}$, while terrestrial nuclear experiments can determine the behavior of matter with good accuracy only up to roughly $10^{11} \mathrm{~g} / \mathrm{cm}^{3}$. Theorists try to fill the large gap between these two densities, but their models suffer from large uncertainties. One of the major goals of neutron star observations is to constrain these uncertainties. A recent effort in this direction is the Neutron star Interior Composition Explorer (NICER), which can infer a neutron star's star mass and radius from x-ray observations of hot spots on the stellar surface (see Sizing Up the Most Massive Neutron Star). Another powerful channel for astrophysical observations involves gravitational waves. In 2017, the LIGO and Virgo collaborations observed a gravitational-wave signal-named GW170817-that originated from a pair of merging neutron stars (see Viewpoint: Neutron Star Merger Seen and Heard). From the data, researchers determined the neutron stars' tidal Love numbers, which-roughly speaking-characterize the shape rigidity of the bodies under tidal forces (see Synopsis: Rising Tides on Black Holes).

One way to extract further information from the different 


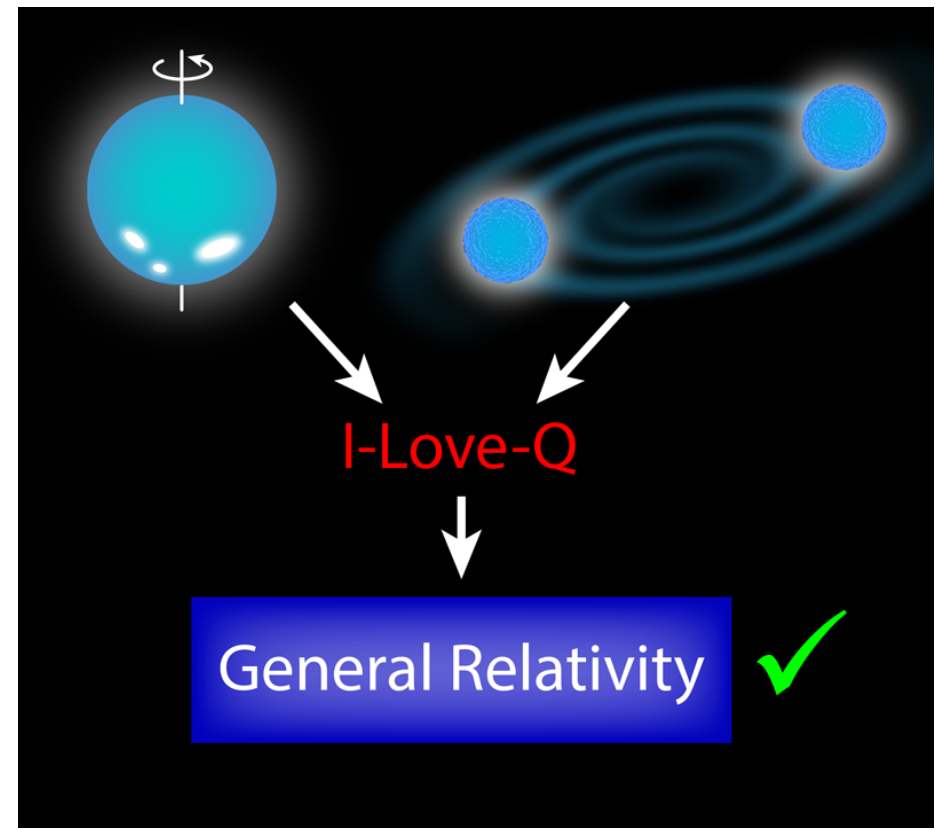

Figure 1: Multimessenger astronomy for neutron stars can consist of x-ray observations of hot spots (left) and gravitational-wave detections from mergers (right). By combining this data and with the help of the so-called universal relations (such as the well-known "I-Love-Q" relations), researchers have been able to test Einstein's theory of general relativity under strong gravity conditions.

Credit: APS/Alan Stonebraker

neutron star observations is through the so-called universal relations, which became popular in the past few years [4-6]. These relations are constructed by computing neutron star properties with a wide range of different nuclear-matter equations of state. Although these equations of state can lead to large differences in the neutron star structure, they do show a consistency in their predictions of different relations, such as one connecting the moment of inertia to the tidal Love number. Because the inferred relations have the "luxury" of being almost independent of the nuclear matter model, they can be used to analyze astrophysical observations of neutron stars [1, 2]. Silva and collaborators built on such universal relations, connecting the neutron star compactness (the ratio of the neutron star mass to its radius) to several parameters that are, roughly speaking, related to the star's mass distribution. The team used compactness values derived from two independent analyses of NICER observational data of the isolated pulsar PSR
J0030+0451 $[7,8]$. Combining the results for the neutron star compactness with the appropriate universal relations, they managed to infer the moment of inertia (I), quadrupole moment $(Q)$, and the surface eccentricity of PSR J0030+0451, which marks the first time that these parameters have been determined for an isolated neutron star. They also demonstrated that the results were only weakly dependent on the employed data-analysis approach.

Using the results for PSR J0030+0451, the researchers also estimated the moment of inertia of PSR J0737-3039A, the larger partner of the only known double pulsar. Ongoing pulsar timing observations of this object will provide an independent estimate of its moment of inertia within the next few years [ 9 , 10]. The possibility to determine the same quantity twice via completely different observations will allow for another test of nuclear matter at extreme densities as well as of strong gravity.

Lastly, Silva and collaborators performed a multimessenger test of general relativity using the moment of inertia of PSR J0030+0451 and the tidal deformability obtained from GW170817 (Fig. 1). For their test, the researchers used another equation-of-state-independent relation-part of the famous I-Love-Q trio [4] -that connects the normalized neutron star moment of inertia and tidal Love number. This universal relation holds not only in general relativity, but also for various alternative theories of gravity (albeit with differences in the exact polynomial dependence connecting the normalized moment of inertia and tidal Love number). By independently inferring the moment of inertia (from the NICER data) and the tidal deformability (from the LIGO/Virgo data) the authors confirmed that Einstein's theory is in agreement with the observations and showed that only a small deviation is allowed. This outcome helped them place strong constraints on parity violation in the gravitational interaction, improving the previous bounds by 7 orders of magnitude.

The research of Silva and collaborators is, on one hand, a culmination of the efforts to use the universal relations to constrain strong gravity using multimessenger astrophysical observations. On the other hand, it opens a new door toward further understanding nuclear matter at extreme densities and testing the laws of gravity. We can expect that such knowledge will soon expand further with the rapid advance of observations. We live in an exciting era when, finally, highly 
accurate instruments are available both in the electromagnetic and in the gravitational-wave channel, allowing us to explore fundamental physics concepts that were considered purely theoretical just a few decades ago. Stay tuned-multimessenger astrophysics will soon bring us new surprises that may challenge our understanding of nature.

Daniela Doneva: Theoretical Astrophysics, Eberhard Karls University of Tübingen, Tübingen, Germany

\section{REFERENCES}

1. K. Yagi and N. Yunes, "Approximate universal relations for neutron stars and quark stars," Phys. Rep. 681, 1 (2017).

2. D. D. Doneva and G. Pappas, "Universal relations and alternative gravity theories," Astrophys. Space Sci. Libr. 457, 737 (2018).

3. H. O. Silva et al., "Astrophysical and theoretical physics implications from multimessenger neutron star observations," Phys. Rev. Lett. 126, 181101 (2021).
4. K. Yagi and N. Yunes, "I-Love-Q: Unexpected universal relations for neutron stars and quark stars," Science 341, 365 (2013).

5. M. Bauböck et al., "Relations between neutron-star parameters in the Hartle-Thorne approximation," Astrophys. J. 777,68 (2013).

6. C. Breu and L. Rezzolla, "Maximum mass, moment of inertia and compactness of relativistic stars," Mon. Not. Roy. Astron. Soc. 459, 646 (2016).

7. T. E. Riley et al., "A NICER view of PSR J0030+0451: Millisecond pulsar parameter estimation," Astrophys. J. Lett 887, L21 (2019).

8. M. C. Miller et al., "PSR J0030+0451 mass and radius from NICER data and implications for the properties of neutron star matter," Astrophys. J. Lett. 887, L24 (2019).

9. J. M. Lattimer and B. F. Schutz, "Constraining the equation of state with moment of inertia measurements," Astrophys. J. 629, 979 (2005).

10. M. Kramer and N. Wex, "The double pulsar system: A unique laboratory for gravity," Class. Quant. Grav. 26, 073001 (2009). 